\title{
ADDITIVE CONJUGACY AND THE BOHR COMPACTIFICATION OF ORTHOGONAL REPRESENTATIONS
}

\author{
ZACHARY CHASE, WADE HANN-CARUTHERS, AND OMER TAMUZ
}

\begin{abstract}
We say that two unitary or orthogonal representations of a finitely generated group $G$ are additive conjugates if they are intertwined by an additive map, which need not be continuous. We associate to each representation of $G$ a topological action that is a complete additive conjugacy invariant: the action of $G$ by group automorphisms on the Bohr compactification of the underlying Hilbert space. Using this construction we show that the property of having almost invariant vectors is an additive conjugacy invariant. As an application we show that $G$ is amenable if and only if there is a nonzero homomorphism from $L^{2}(G)$ into $\mathbb{R} / \mathbb{Z}$ that is invariant to the $G$-action.
\end{abstract}

\section{InTRODUCTION}

Let $G$ be a finitely generated group. To each unitary or orthogonal representation of $G$ one can associate a probability measure preserving actionthe so-called Gaussian action. Conversely, to each probability measure preserving action of $G$ one can associate the Koopman representation. These constructions have proven to be an important connection between ergodic theory and representation theory, with many interesting applications (see, e.g., $[2,3,7,8])$.

In this paper we associate a topological action to representations, with the goal of establishing connections between the dynamical properties of the representation and the action. We use this construction to study properties of the representation that are "additive conjugacy" invariants; we define this notion below. As an application we derive a new characterization of amenability.

Omer Tamuz was supported by a grant from the Simons Foundation (\#419427), a Sloan research fellowship, a BSF award (\#2018397), and an NSF CAREER award (DMS1944153). 
Let $G \curvearrowright^{\pi} \mathcal{H}$ be an orthogonal representation of a finitely generated group on a separable real Hilbert space. ${ }^{1}$ We associate to the representation $\pi$ the topological action of $G$ on the Bohr compactification of $\mathcal{H}$. In general, the Bohr compactification $b A$ of a topological abelian group $A$ is the algebraic dual of $\hat{A}_{d}$, where the latter is the algebraic dual of $A$, equipped with the discrete topology. As we explain in $§ 2.2$, in our case of a real separable Hilbert space $\mathcal{H}$, an equivalent definition is to let $b \mathcal{H}$ be the set of all homomorphisms (i.e., additive maps) from $\mathcal{H}$ to $\mathbb{T}=\mathbb{R} / \mathbb{Z}$ :

$$
b \mathcal{H}=\{\varphi: \mathcal{H} \rightarrow \mathbb{T} \mid \varphi(v+w)=\varphi(v)+\varphi(w)\} .
$$

Importantly, $b \mathcal{H}$ includes maps that are not continuous. The Bohr compactification $b \mathcal{H}$ is indeed compact, when endowed with the subspace topology induced from the product space $\mathbb{T}^{\mathcal{H}}$. It also admits the obvious abelian group structure, which is compatible with this topology.

The group $G$ acts on $b \mathcal{H}$ by precomposition:

$$
[g \varphi](v)=\varphi\left(\pi_{g}^{-1} v\right) .
$$

It is straightforward to verify that this action is by automorphisms of $b \mathcal{H}$ as a topological group. Thus the action $G \curvearrowright b \mathcal{H}$ is a topological algebraic action that is associated to the representation $\pi$. This action will be useful in the study of the following notion of conjugacy:

Definition 1. Two representations, $G \curvearrowright^{\pi} \mathcal{H}$ and $G \curvearrowright^{\pi^{\prime}} \mathcal{H}^{\prime}$, are additive conjugates if there exists a bijection $\xi: \mathcal{H} \rightarrow \mathcal{H}^{\prime}$ such that for all $v, w \in \mathcal{H}$ and $g \in G$,

$$
\xi(v+w)=\xi(v)+\xi(w)
$$

and

$$
\xi\left(\pi_{g} v\right)=\pi_{g}^{\prime} \xi(v)
$$

That is, two representations are additive conjugates if they are intertwined by an additive bijection. Note that this bijection need not be continuous.

It is straightforward to check that the action on the Bohr compactification is a complete additive conjugacy invariant. That is, that $\pi$ and $\pi^{\prime}$ are additive conjugates if and only if $G \curvearrowright b \mathcal{H}$ and $G \curvearrowright b \mathcal{H}^{\prime}$ are conjugates, as topological algebraic actions (Claim 8).

\footnotetext{
${ }^{1}$ Note that the class of orthogonal representations includes the unitary ones; we elaborate on this in $\S 2.1$. Thus all of our results apply to unitary representations on separable complex Hilbert spaces.
} 
1.1. Main results. In all of our results below, $G$ is a finitely generated group, Hilbert spaces are separable and either real or complex, and representations are, respectively, either orthogonal or unitary - unless otherwise specified.

Our main result ties an important property of a representation with a dynamical property of its associated topological action. Recall that $\pi$ is said to have almost invariant vectors if there exists a sequence of unit vectors $\left(u_{n}\right)_{n}$ in $\mathcal{H}$ such that $\lim _{n}\left\|\pi_{g} u_{n}-u_{n}\right\|=0$ for each $g \in G$. A fixed point $x$ of a topological action $G \curvearrowright X$ is one that satisfies $g x=x$ for all $g \in G$.

Theorem 1. $G \curvearrowright^{\pi} \mathcal{H}$ has almost invariant vectors if and only if the associated action $G \curvearrowright b \mathcal{H}$ has a nonzero fixed point.

Since the action on the Bohr compactification is a complete additive conjugacy invariant, this theorem has an immediate corollary.

Corollary 2. Let $\pi_{1}$ and $\pi_{2}$ be additive conjugates. Then $\pi_{1}$ has almost invariant vectors if and only if $\pi_{2}$ has almost invariant vectors.

We note that, as far as we know, this is not known even for the case of $G=\mathbb{Z}$. This corollary may be a priori surprising, since almost invariant vectors are defined using the topology of the Hilbert space, whereas this topology does not appear in the definition of additive conjugacy.

By the Hulanicki-Reiter Theorem (see, e.g., [1, Theorem G.3.2]), $G$ is amenable if and only if the left regular real representation $G \curvearrowright^{\lambda} L^{2}(G)$ has almost invariant vectors. Hence the following is another corollary of Theorem 1:

Corollary 3. $G$ is amenable if and only if there exists a nonzero homomorphism $\varphi: L^{2}(G) \rightarrow \mathbb{T}$ that is invariant to the $G$ action: $\varphi(f)=\varphi\left(\lambda_{g} f\right)$ for all $f \in L^{2}(G)$ and $g \in G$.

Note that this homomorphism is not necessarily continuous.

1.2. Proof sketch. Both directions of the proof of Theorem 1 require some work. An important tool is the natural homomorphism $\sigma: \mathcal{H} \rightarrow b \mathcal{H}$, which, for a real Hilbert space $\mathcal{H}$, is given by

$$
\left[\sigma_{v}\right](w)=\langle v, w\rangle+\mathbb{Z}
$$

When $\pi$ has almost invariant vectors $\left(v_{n}\right)_{n}$, it is straightforward to show that any limit point of $\left(\sigma_{v_{n}}\right)_{n}$ is a fixed point of $b \mathcal{H}$. However, this fixed point might be zero. To overcome this, we construct from $\left(v_{n}\right)_{n}$ a modified sequence of almost invariant vectors $\left(w_{n}\right)_{n}$ such that all limit points of $\left(\sigma_{w_{n}}\right)_{n}$ are nonzero. 
When $\pi$ does not have almost invariant vectors, we in fact prove a stronger statement. Given a symmetric probability measure $\mu$ on $G$ whose support is equal to a finite generating set of $G$ containing the identity, we say that $\varphi \in b \mathcal{H}$ is $\mu$-harmonic if

$$
\varphi\left(\sum_{g} \mu(g) \pi_{g} v\right)=\varphi(v)
$$

for every $v \in \mathcal{H}$. We prove the following claim, which implies the corresponding direction of Theorem 1.

Proposition 4. Suppose $\pi$ does not have almost invariant vectors and $\mu$ is a symmetric generating measure for $G$. Then bH has no nonzero $\mu$-harmonic points, and in particular has no nonzero fixed points.

1.3. Open questions and additional results. This paper leaves unanswered the larger question of what properties of a representation are reflected in its Bohr compactification, or, equivalently, what properties are additive conjugacy invariants.

In $§ 5$ we completely classify the additive conjugacy classes of the irreducible unitary representations of $\mathbb{Z}$, showing that these are determined by the Galois conjugacy class of the eigenvalue. In particular, the case of $\mathbb{Z}$ shows that representations that are not conjugate (in the the usual sense) can be additive conjugates, and that furthermore this holds even for irreducible representations. A similar analysis should apply to $\mathbb{Z}^{d}$. For other groups, we leave this question for future research.

One may imagine that there is some connection between weak containment and additive conjugacy. Indeed, this is perhaps suggested by Corollary 2. We prove an additional result in this direction. This result can be interpreted to imply that the Bohr compactification records the data of the weakly contained irreducible representations.

Proposition 5. Let $G$ be a finitely generated group. Let $G \curvearrowright^{\pi} \mathcal{H}$ be an orthogonal representation that weakly contains the irreducible orthogonal representation $G \curvearrowright^{\pi^{\prime}} \mathcal{H}^{\prime}$. Then for every $v^{\prime} \in \mathcal{H}^{\prime}$ there are $\varphi \in b \mathcal{H}$ and $v \in \mathcal{H}$ such that for all $g \in G$,

$$
[g \varphi](v)=\left\langle\pi_{g}^{\prime} v^{\prime}, v^{\prime}\right\rangle+\mathbb{Z} .
$$

This proposition, together with Theorem 1, suggests the following (perhaps bold) conjecture.

Conjecture 6. Let $G$ be a finitely generated group. Let $G \curvearrowright^{\pi} \mathcal{H}$ be an orthogonal representation. Then the following are equivalent: 
(1) $\pi$ weakly contains the irreducible orthogonal representation $G \curvearrowright^{\pi^{\prime}}$ $\mathcal{H}^{\prime}$.

(2) There is a closed, $G$-invariant subgroup $K \subseteq$ bH such that the topological algebraic actions $G \curvearrowright K$ and $G \curvearrowright b \mathcal{H}^{\prime}$ are conjugate.

Acknowledgments. We would like to thank Todor Tsankov for suggesting some improvements to our proofs, Yehuda Shalom for suggesting to us the classification of the additive conjugacy classes of the irreducible representations of $\mathbb{Z}$, and Andreas Thom for pointing out an error in an earlier version, as well as suggesting a correct proof. We would also like to thank Joshua Frisch, Eli Glasner, Alexander Kechris, Jesse Peterson, Pooya Vahidi Ferdowsi, Benjamin Weiss, and Andy Zucker for helpful discussions.

\section{Definitions}

2.1. Orthogonal and unitary representations. Let $\mathcal{H}$ be a separable real Hilbert space equipped with an inner product $\langle\cdot, \cdot\rangle$. An orthogonal representation $\pi$ of a discrete group $G$ is a homomorphism $\pi: G \rightarrow O(\mathcal{H})$, where $O(\mathcal{H})$ is the group of orthogonal (i.e., linear and inner product preserving) bijections from $\mathcal{H}$ to $\mathcal{H}$. That is, $\pi$ is a group homomorphism of $G$ into the group of linear transformations of $\mathcal{H}$ that preserve the inner product of $\mathcal{H}$. We henceforth omit $\pi$ from our notation and write the image of $w \in \mathcal{H}$ under $\pi_{g}$ simply as $g w$.

As the next lemma shows, every unitary representation on a complex Hilbert space $\mathcal{H}$ is also an orthogonal representation of the associated real Hilbert space.

Lemma 7. Let $G \curvearrowright^{\pi} \mathcal{H}$ be a unitary representation of $G$ on a complex Hilbert space $\mathcal{H}$. Let $\widetilde{\mathcal{H}}$ denote the realification of $\mathcal{H}$, with inner product $\langle u, v\rangle_{\widetilde{\mathcal{H}}}:=\Re\langle u, v\rangle_{\mathcal{H}}$. Let $\widetilde{\pi}: G \curvearrowright \widetilde{\mathcal{H}}$ be the same action as $\pi$. Then $\widetilde{\pi}$ is an orthogonal representation, and $\pi$ has almost invariant vectors if and only if $\tilde{\pi}$ does.

Proof. For any $v, w \in \widetilde{\mathcal{H}},\langle g v, g w\rangle_{\widetilde{\mathcal{H}}}=\Re\langle g v, g w\rangle_{\mathcal{H}}=\Re\langle v, w\rangle_{\mathcal{H}}=\langle v, w\rangle_{\widetilde{\mathcal{H}}}$. The equivalence of having almost invariant vectors follows from the fact that the norms on $\mathcal{H}$ and $\widetilde{\mathcal{H}}$ are the same and that the actions are the same.

It follows from Lemma 7 that it suffices to prove Theorem 1 for orthogonal representations to conclude that it also holds for unitary representations. The same holds for Corollary 2 and Proposition 4. 
2.2. Pontryagin duality and the Bohr compactification. A homomorphism of a topological abelian group $A$ into $\mathbb{T}=\mathbb{R} / \mathbb{Z}$ is a map $\varphi$ that satisfies $\varphi(v+w)=\varphi(v)+\varphi(w)$ for all $v, w \in A$. The set of all continuous such homomorphisms, equipped with the compact-open topology, is called the algebraic dual of $A$ and is denoted by $\hat{A}$. The Bohr compactification $b A$ of $A$ is $\widehat{\hat{A}}_{d}$, where $\hat{A}_{d}$ is $\hat{A}$ equipped with the discrete topology. That is, $b A$ is the set of all (i.e., not necessarily continuous) homomorphisms from $\hat{A}$ to $\mathbb{T}$.

The natural map $\tau: A \rightarrow b A$ given by

$$
[\tau(v)](\gamma)=\gamma(v)
$$

is known to be injective and continuous when $A$ is locally compact, in which case its image is dense in $b A$.

Some groups, such as $\mathbb{R}$, can be (non-canonically) identified with their algebraic dual. In this case, $b A$ is simply the set of all homomorphisms from $A$ to $\mathbb{T}$. As we show (Proposition 15) this identification holds for a separable real Hilbert space $\mathcal{H}$. We hence define the Bohr compactification of $\mathcal{H}$ as in (1), by letting $b \mathcal{H}$ be the algebraic dual of $\mathcal{H}$ equipped with the discrete topology.

Since $b \mathcal{H}$ is compact, it follows from the Pontryagin Duality Theorem (see, e.g., [5]) that its algebraic dual $\widehat{b \mathcal{H}}$ can be canonically identified with $\mathcal{H}$ equipped with the discrete topology. This identification is realized by

$$
v(\varphi)=\varphi(v) .
$$

2.3. Generating measures and harmonic homomorphisms. Let $S$ be a finite, symmetric generating set for $G$ containing the identity, and let $\mu$ be a symmetric probability measure whose support is equal to $S$. We call such $\mu$ "symmetric generating measures".

Let $P_{\mu}: \mathcal{H} \rightarrow \mathcal{H}$ be the continuous linear operator given by

$$
P_{\mu} w=\sum_{h \in S} \mu(h) h w,
$$

and let $D_{u}: \mathcal{H} \rightarrow \mathcal{H}$ be given by

$$
D_{\mu} w=w-P_{\mu} w .
$$

We say that $\varphi \in b \mathcal{H}$ is $\mu$-harmonic if $\varphi\left(D_{\mu} w\right)=0$ for all $w \in \mathcal{H}$. By additivity, this is equivalent to the definition above, in (2). 


\section{General properties of the action on the Bohr COMPACTIFICATION}

In this section we outline some simple, general properties of the action on the Bohr compactification and its relation to the representation.

Given a compact group $A$, an algebraic action $G \curvearrowright A$ is a homomorphism from $G$ into the group of continuous group isomorphisms of $A$. Thus, two algebraic actions are conjugate if they are intertwined by a continuous group isomorphism.

Claim 8. Two orthogonal representations of $G \curvearrowright^{\pi} \mathcal{H}$ and $G \curvearrowright^{\pi^{\prime}} \mathcal{H}^{\prime}$ are additive conjugates if and only if $G \curvearrowright b \mathcal{H}$ and $G \curvearrowright b \mathcal{H}^{\prime}$ are conjugate algebraic actions.

Proof. Assume first that $\xi: \mathcal{H} \rightarrow \mathcal{H}^{\prime}$ witnesses the additive conjugacy of $\pi$ and $\pi^{\prime}$. Then $\mathcal{H}$ and $\mathcal{H}^{\prime}$ are isomorphic as discrete abelian groups, and hence their algebraic duals $b \mathcal{H}$ and $b \mathcal{H}^{\prime}$ are isomorphic as topological groups; this is witnessed by $\xi_{*}: b \mathcal{H} \rightarrow b \mathcal{H}^{\prime}$, defined by

$$
\left[\xi_{*} \varphi\right]\left(v^{\prime}\right)=\varphi\left(\xi^{-1} v^{\prime}\right)
$$

It is straightforward to check that since $\xi$ intertwines $\pi$ and $\pi^{\prime}$, it holds that $\xi_{*} g \varphi=g \xi_{*} \varphi$, and hence the actions $G \curvearrowright b \mathcal{H}$ and $G \curvearrowright b \mathcal{H}^{\prime}$ are conjugate.

Conversely, assume that $\xi_{*}: b \mathcal{H} \rightarrow b \mathcal{H}^{\prime}$ witnesses the conjugacy of $G \curvearrowright$ $b \mathcal{H}$ and $G \curvearrowright b \mathcal{H}^{\prime}$. Then in particular $b \mathcal{H}$ and $b \mathcal{H}^{\prime}$ are isomorphic as topological groups, and hence their algebraic duals $\mathcal{H}$ and $\mathcal{H}^{\prime}$ - endowed with the discrete topology - are conjugate (see the end of $\S 2.2$ ). This is witnessed by $\xi: \mathcal{H} \rightarrow \mathcal{H}^{\prime}$, defined by

$$
[\xi v]\left(\varphi^{\prime}\right)=v\left(\xi_{*}^{-1} \varphi\right)
$$

It is again straightforward to check that since $\xi_{*}$ intertwines the actions on $b \mathcal{H}$ and $b \mathcal{H}^{\prime}, \xi$ intertwines $\pi$ and $\pi^{\prime}$. Likewise, $\xi$ is immediately seen to be additive.

Let $m$ be the unique Haar probability measure on the compact group $b \mathcal{H}$. Since $G$ acts on $b \mathcal{H}$ by automorphisms, it preserves $m$, and so $G \curvearrowright(b \mathcal{H}, m)$ is a probability measure preserving action.

Claim 9. The following are equivalent:

(1) The action $G \curvearrowright(b \mathcal{H}, m)$ is ergodic.

(2) The action $G \curvearrowright b \mathcal{H}$ is topologically transitive.

(3) The orbit $\{g v \mid g \in G\}$ is infinite for every nonzero $v \in \mathcal{H}$. 
Proof. The first two conditions are equivalent by [9, Theorem 1.1] (in fact, this equivalence holds more generally for actions by automorphisms on compact groups). By a result of Halmos [4] for $\mathbb{Z}$ actions, which was extended by Kaplansky to finitely generated groups $[6],{ }^{2}$ non-ergodicity of the action $G \curvearrowright(b \mathcal{H}, m)$ is equivalent to the existence of a nonzero $\chi \in \widehat{b \mathcal{H}}$ with a finite $G$-orbit. It follows from Pontryagin duality that the dual $\widehat{b \mathcal{H}}$ of $b \mathcal{H}$ can be identified with $\mathcal{H}$, equipped with the discrete topology (see the end of $\S 2.2$ ). Thus a nonzero character $\chi \in \widehat{b \mathcal{H}}$ with a finite orbit is simply equivalent to a nonzero vector $v \in \mathcal{H}$ with a finite orbit.

\section{Proofs}

\subsection{Preliminary claims.}

Claim 10. Every fixed point of bH is $\mu$-harmonic for every symmetric generating measure $\mu$.

Proof. Suppose $\mu$ is a symmetric generating measure with support $S$ and $\varphi$ is a fixed point of $b \mathcal{H}$. Then for any $v \in H$,

$$
\varphi\left(D_{\mu} v\right)=\varphi\left(v-\sum_{g \in S} \mu(g) g v\right)=\varphi(v)-\sum_{g \in S} \varphi(g(\mu(g) v)) .
$$

Because $\varphi$ is a fixed point, $\varphi(g(\mu(g) v))=\varphi(\mu(g) v)$. Hence,

$$
\varphi\left(D_{\mu} v\right)=\varphi(v)-\sum_{g \in S} \varphi(\mu(g) v)=\varphi(v)-\varphi\left(\sum_{g \in S} \mu(g) v\right) .
$$

But $\mu$ is a probability measure, and so $\sum \mu(g) v=v$. We thus obtain

$$
\varphi\left(D_{\mu} v\right)=\varphi(v)-\varphi(v)=0 .
$$

\subsection{Proof of main theorem.}

Lemma 11. Suppose $\pi$ does not have a nonzero invariant vector. Then if it has almost invariant vectors, it has almost invariant vectors that are mutually orthogonal.

Note that the assumptions of the lemma imply that the Hilbert space is infinite dimensional. In finite dimensional spaces, whenever there are almost invariant vectors there are non-zero invariant vectors, since the unit sphere is compact.

\footnotetext{
${ }^{2}$ See also [9, Lemma 1.2 and remark (3) on page 9].
} 
Proof of Lemma 11. Let $\left(v_{n}\right)_{n}$ be a sequence of almost invariant vectors. Since the unit ball is weakly sequentially compact, there is some subsequence $\left(v_{n_{k}}\right)_{k}$ so that $v_{n_{k}} \rightarrow v$ for some $\|v\| \leq 1$. For ease, relabel $\left(v_{n_{k}}\right)_{k}=\left(v_{n}\right)_{n}$. We show $v$ is $G$-invariant: for any $g \in G$, we have $g v_{n} \rightarrow g v$ and so $g v_{n}-v_{n} \rightarrow g v-v$. Since $g v_{n}-v_{n} \rightarrow 0$, we must have $g v-v=0$. By assumption, $v$ must be equal to 0 . Thus $\left(v_{n}\right)_{n}$ is a sequence of almost invariant (unit) vectors that weakly converge to 0 .

We construct a slightly altered sequence $\left(w_{k}\right)_{k}$ of mutually orthogonal vectors that are still almost-invariant. Let $w_{1}=v_{1}$. With $w_{1}, \ldots, w_{k}$ chosen, choose $m$ large enough so that $m>k$ and

$$
\left|\left\langle v_{m}, w_{i}\right\rangle\right|<\frac{1}{k}
$$

for $1 \leq i \leq k$, which is possible since, for each $1 \leq i \leq k,\left\langle w_{i}, v_{m}\right\rangle \rightarrow 0$ as $m \rightarrow \infty$. Let $\hat{v}_{m}$ denote the projection of $v_{m}$ onto the linear space spanned by $w_{1}, \ldots, w_{k}$, and note that

$$
\left\|\hat{v}_{m}\right\|^{2}=\sum_{i=1}^{k}\left|\left\langle v_{m}, w_{i}\right\rangle\right|^{2}<\frac{1}{k} .
$$

Let

$$
w_{k+1}=\frac{v_{m}-\hat{v}_{m}}{\left\|v_{m}-\hat{v}_{m}\right\|} .
$$

By construction the $w_{k}$ 's are mutually orthogonal, each with norm 1 . Observe that for any $g \in G$,

$$
\begin{aligned}
\left\|g w_{k+1}-w_{k+1}\right\| & =\frac{1}{\left\|v_{m}-\hat{v}_{m}\right\|}\left\|g v_{m}-v_{m}+\hat{v}_{m}-g \hat{v}_{m}\right\| \\
& \leq \frac{1}{1-1 / \sqrt{k}}\left[\left\|g v_{m}-v_{m}\right\|+2\left\|\hat{v}_{m}\right\|\right] \\
& \leq \frac{1}{1-1 / \sqrt{k}}\left[\left\|g v_{m}-v_{m}\right\|+\frac{2}{\sqrt{k}}\right] .
\end{aligned}
$$

Thus, since $m>k,\left(w_{k}\right)_{k}$ is an almost invariant sequence.

Proposition 12. If $\pi$ has almost invariant vectors, then $b \mathcal{H}$ has a nonzero fixed point.

Proof. If $\pi$ has a nonzero invariant vector, say $w$, then $\sigma_{w}$ is clearly a nonzero fixed point of $b \mathcal{H}$. So we may suppose $\pi$ has no nonzero invariant vector. Then, by Lemma 11, we may let $\left(v_{n}\right)_{n}$ be a sequence of almost invariant (unit) vectors such that for all $m \neq n,\left\langle v_{m}, v_{n}\right\rangle=0$. 
Fix a finite generating set $S$ for $G$. By passing to a subsequence of $\left(v_{n}\right)_{n}$ if necessary, we may suppose that for all $n \geq 1$ and all $h \in S$, we have

$$
\left\|h v_{n}-v_{n}\right\|<\frac{1}{2^{n}} \text {. }
$$

For each $n \geq 1$, let

$$
\epsilon_{n}=\max _{h \in S}\left\|h v_{n}-v_{n}\right\|
$$

and define

$$
w_{n}=\frac{v_{n}}{\sqrt{\epsilon_{n}}} .
$$

Since $b \mathcal{H}$ is compact, there is a subnet $\left(w_{\alpha}\right)_{\alpha}$ of $\left(w_{n}\right)_{n}$ so that $\left(\sigma_{w_{\alpha}}\right)_{\alpha}$ converges, say to $\varphi \in b \mathcal{H}$.

We first show $\varphi \neq 0$. To this end, let

$$
w=\frac{1}{2} \sum_{n=1}^{\infty} \epsilon_{n} w_{n}
$$

which is well-defined since

$$
\sum_{n=1}^{\infty}\left|\epsilon_{n} w_{n}\right|=\sum_{n=1}^{\infty} \sqrt{\epsilon_{n}}<\sum_{n=1}^{\infty} \sqrt{2^{-n}}<\infty .
$$

Note, for any $n \geq 1$,

$$
\left\langle w, w_{n}\right\rangle=\frac{1}{2} \epsilon_{n}\left\langle w_{n}, w_{n}\right\rangle=\frac{1}{2}
$$

by orthogonality. Therefore,

$$
\varphi(w)=\lim _{\alpha}\left\langle w_{\alpha}, w\right\rangle+\mathbb{Z}=\frac{1}{2}+\mathbb{Z}
$$

shows indeed $\varphi \neq 0$.

We finish by showing $\varphi$ is a fixed point of $b \mathcal{H}$. Take $v \in \mathcal{H}$ and $h \in S$. Then

$$
\varphi\left(h^{-1} v\right)-\varphi(v)=\lim _{\alpha}\left(\sigma_{w_{\alpha}}\left(h^{-1} v\right)-\sigma_{w_{\alpha}}(v)\right)=\lim _{\alpha}\left(\left\langle v, h w_{\alpha}\right\rangle-\left\langle v, w_{\alpha}\right\rangle+\mathbb{Z}\right) .
$$

Now,

$$
\begin{aligned}
\lim _{\alpha}\left|\left\langle v, h w_{\alpha}\right\rangle-\left\langle v, w_{\alpha}\right\rangle\right| & =\lim _{\alpha}\left|\left\langle v, h w_{\alpha}-w_{\alpha}\right\rangle\right| \\
& \leq \lim _{\alpha}\|v\|\left\|h w_{\alpha}-w_{\alpha}\right\| .
\end{aligned}
$$

Since

$$
\left\|h w_{n}-w_{n}\right\| \leq \sqrt{\epsilon_{n}} \rightarrow 0
$$


it holds that

$$
\left\|h w_{\alpha}-w_{\alpha}\right\| \rightarrow 0
$$

and therefore we see $\varphi\left(h^{-1} v\right)=\varphi(v)$ for each $h \in S$. Since $S$ is a symmetric generating set, $\varphi$ is a fixed point of $b \mathcal{H}$.

The folloming lemma is standard; we omit the proof. We will be use it to show $D_{\mu}$ is surjective.

Lemma 13. Let $T: \mathcal{H} \rightarrow \mathcal{H}$ be a bounded linear operator. Suppose there exists some $c>0$ so that $\|T x\| \geq c\|x\|$ for all $x \in H$. Then the range of $T$ is closed.

Proposition 14. If $\pi$ does not have almost invariant vectors, then $D_{\mu}$ is surjective for any symmetric generating measure $\mu$.

Proof. First observe that $D_{\mu}$ is self-adjoint since $\mu$ is symmetric. Furthermore, $D_{\mu}$ is injective since there are no nonzero invariant vectors. Indeed, note that if $D_{\mu}(w)=0$ for some unit vector $w$, then

$$
w=\sum_{h} \mu(h) h w
$$

and so

$$
1=\sum_{h} \mu(h)\langle h w, w\rangle .
$$

The right hand side is the average of numbers that are at most 1 . Since this average is equal to 1 they all have to equal 1 , and so (since $\mu$ is generating) $w$ is invariant.

Since $D_{\mu}$ is self-adjoint and injective, $D_{\mu}$ has a dense image. Hence, by Lemma 13, it suffices to show the lower bound inequality. Since, for $\|v\|=1$,

$$
\left\|D_{\mu}(v)\right\|=\left\|v-P_{\mu}(v)\right\| \geq\|v\|-\left\|P_{\mu}(v)\right\|=1-\left\|P_{\mu}(v)\right\|,
$$

it suffices to bound $\left\|P_{\mu}(v)\right\|$ away from 1 . This is an immediate consequence of [1, Proposition 6.2.1], but we include a proof for completeness.

Let $S$ denote the support of $\mu$. Since there are no almost invariant vectors, there is an $\epsilon>0$ so that for all $\|v\|=1$ there exists an $h \in S$ such that $\|h v-v\| \geq \epsilon$ (see, e.g., [1, Proposition F.1.7]). Note for such a $v$ and $h$, we have

$$
\epsilon^{2} \leq\|h v-v\|^{2}=\langle h v-v, h v-v\rangle=1-2\langle v, h v\rangle+1
$$

and thus

$$
\langle v, h v\rangle \leq 1-\frac{1}{2} \epsilon^{2} .
$$


Therefore, for any unit vector $v \in \mathcal{H}$, taking again $h_{0} \in S$ so that $\| h_{0} v-$ $v \| \geq \epsilon$ gives

$$
\begin{aligned}
\left\|P_{\mu} v\right\|^{2} & =\left\langle\sum_{h \in S} \mu(h) h v, \sum_{h^{\prime} \in S} \mu\left(h^{\prime}\right) h^{\prime} v\right\rangle \\
& =\mu\left(h_{0}\right) \mu(e)\left\langle h_{0} v, v\right\rangle+\sum_{\left(h, h^{\prime}\right) \neq\left(h_{0}, e\right)} \mu(h) \mu\left(h^{\prime}\right)\left\langle h v, h^{\prime} v\right\rangle \\
& \leq \mu\left(h_{0}\right) \mu(e)\left(1-\frac{1}{2} \epsilon^{2}\right)+\sum_{\left(h, h^{\prime}\right) \neq\left(h_{0}, e\right)} \mu(h) \mu\left(h^{\prime}\right) \\
& =1-\frac{1}{2} \mu\left(h_{0}\right) \mu(e) \epsilon^{2} .
\end{aligned}
$$

Consequently, for each $\|v\|=1$,

$$
\left\|P_{\mu} v\right\|^{2} \leq 1-\frac{1}{2} \epsilon^{2} \mu(e) \inf _{h \in S} \mu(h) .
$$

Since $S$ is finite, we are done.

We now have the tools to prove Proposition 4.

Proof of Proposition 4. Suppose $\pi$ does not have almost invariant vectors, $\mu$ is a symmetric generating measure, and $\varphi$ is a $\mu$-harmonic point of $b \mathcal{H}$. Then $\varphi\left(D_{\mu}(\mathcal{H})\right)=0$. Now, by Proposition 14 , since $\pi$ does not have almost invariant vectors, $D_{\mu}$ is surjective. Hence, $\varphi(\mathcal{H})=0$, and so $\varphi=0$. Thus, the only $\mu$-harmonic point of $b \mathcal{H}$ is 0 . Further, since every fixed point of $b \mathcal{H}$ is $\mu$-harmonic (Claim 10), it follows that the only fixed point of $b \mathcal{H}$ is 0 .

We are now ready to prove our main theorem.

Proof of Theorem 1. First, suppose that $\pi$ has almost invariant vectors. Then by Proposition $12, b \mathcal{H}$ has a nonzero fixed point. Now, suppose that $b \mathcal{H}$ has a nonzero fixed point. Then by Proposition $4, \pi$ must have almost invariant vectors.

4.3. The Bohr compactification of a separable Hilbert space. Let $\mathcal{H}$ be a separable real Hilbert space. Recall that the algebraic dual of $\mathcal{H}$ is given by

$$
\widehat{\mathcal{H}}=\{\phi: \mathcal{H} \rightarrow \mathbb{T} \mid \phi \text { is a continuous homomorphism }\}
$$

As above, for each $v \in \mathcal{H}$, let $\sigma_{v}: \mathcal{H} \rightarrow \mathbb{T}$ be given by

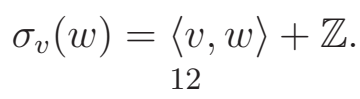


Proposition 15. A real separable Hilbert space $\mathcal{H}$, equipped with the weak topology, can be identified as a topological group with its algebraic dual $\widehat{\mathcal{H}}$ via $v \mapsto \sigma_{v}$.

Proof of Proposition 15. We first note that every $\sigma_{v}$ is an element of $\widehat{\mathcal{H}}$, i.e., is a continuous homomorphism from $\mathcal{H}$ to $\mathbb{T}$. This follows from the fact that $w \mapsto\langle v, w\rangle$ is weakly continuous, and that the projection $\mathbb{R} \rightarrow \mathbb{T}$ is also continuous. We now show that every continuous homomorphism $\phi: \mathcal{H} \rightarrow \mathbb{T}$ is equal to some $\sigma_{w}$.

Given $\phi$, let $\psi: \mathcal{H} \rightarrow \mathbb{R}$ be the unique lift of $\phi$ satisfying $\psi(0)=0$. Then $\psi$ is continuous and is easily seen to furthermore be linear. Hence it must be of the form $\psi(v)=\langle v, w\rangle$ for some $w \in \mathcal{H}$. Since $\psi$ is a lift of $\phi$, $\phi(v)=\langle v, w\rangle+\mathbb{Z}$. We have thus shown that $\phi=\sigma_{w}$.

Finally, we argue that $v \mapsto \sigma_{v}$ is continuous and has a continuous inverse. It follows immediately from the definition that if a net $\left(v_{\alpha}\right)_{\alpha}$ converges weakly to $v$ then $\left(\sigma_{v_{\alpha}}\right)_{\alpha}$ converges to $\sigma_{v}$. Hence $v \mapsto \sigma_{v}$ is continuous. Since the unit ball in $\mathcal{H}$ is weakly compact, the restriction of this map to this ball has an inverse that is also continuous. By the additivity of the map $v \mapsto \sigma_{v}$, it follows that this map has a continuous inverse.

\subsection{Proof of Proposition 5.}

Lemma 16. Let $\rho: G \rightarrow \mathbb{T}$ be a function. Suppose there exist vectors $\left(v_{n}\right)_{n}$ that weakly converge to 0 , and that for each $g \in G, \lim _{n}\left\langle g v_{n}, v_{n}\right\rangle+\mathbb{Z}=\rho(g)$. Then there exist $\varphi \in b \mathcal{H}$ and $v \in \mathcal{H}$ so that $\varphi\left(g^{-1} v\right)=\rho(g)$ for each $g \in G$.

Proof. Let $\left\{g_{1}, g_{2}, \ldots\right\}$ be an enumeration of (the countable group) $G$. Let $w_{1}=v_{1}$. With $w_{1}, \ldots, w_{n-1}$ chosen, let $w_{n}=v_{k_{n}}$ be such that $\left|\left\langle v_{k_{n}}, g w_{j}\right\rangle\right|<$ $2^{-n^{2}}$ for each $1 \leq j \leq n-1$ and $g \in\left\{g_{1}, g_{1}^{-1}, \ldots, g_{n-1}, g_{n-1}^{-1}\right\}$, which is possible since $v_{n} \rightarrow 0$. Let $v=\sum_{n} 2^{-n} w_{n}$. As $b \mathcal{H}$ is compact, we may take $\varphi$, a limit of some subnet of $\left(2^{n} \sigma_{w_{n}}\right)_{n}$. Fix $g \in G$. Note, for each $n \geq 1$,

$$
2^{n} \sigma_{w_{n}}\left(g^{-1} v\right)=\left\langle g w_{n}, w_{n}\right\rangle+\sum_{k<n} 2^{n} 2^{-k}\left\langle g^{-1} w_{k}, w_{n}\right\rangle+\sum_{k>n} 2^{n} 2^{-k}\left\langle w_{k}, g w_{n}\right\rangle .
$$

By construction, if $g=g_{l}$ and $n>l$, then for $k<n,\left|\left\langle g^{-1} w_{k}, w_{n}\right\rangle\right| \leq 2^{-n^{2}}$ and for $k>n,\left|\left\langle w_{k}, g w_{n}\right\rangle\right| \leq 2^{-k^{2}}$. So, we obtain that

$$
\begin{aligned}
\lim _{n} 2^{n} \sigma_{w_{n}}\left(g^{-1} v\right) & =\lim _{n}\left(\left\langle g w_{n}, w_{n}\right\rangle+O\left(n 2^{n} 2^{-n^{2}}+\sum_{k>n} 2^{n-k^{2}}\right)\right) \\
& =\rho(g) .
\end{aligned}
$$

We conclude $\varphi\left(g^{-1} v\right)=\rho(g)$. 
Proof of Proposition 5. Since $\pi$ weakly contains $\pi^{\prime}$ and $\pi^{\prime}$ is irreducible, for any $v^{\prime} \in \mathcal{H}^{\prime}$, there exists a sequence of vectors $\left(v_{n}\right)_{n}$ in $\mathcal{H}$ such that $\lim \left\langle g v_{n}, v_{n}\right\rangle=\left\langle g v^{\prime}, v^{\prime}\right\rangle$ for each $g \in G$ (see, e.g., [1, Proposition F.1.4]). Since the norm of $v_{n}$ converges to the norm of $v^{\prime}$, we may assume that $\left(v_{n}\right)_{n}$ has a weak limit, say $v$. If $v=0$, then the result follows immediately from Lemma 16. So we may assume $v \neq 0$.

For each $n$, let $u_{n}=v_{n}-v$. For any $g \in G$, it follows from $u_{n} \rightarrow 0$ that

$$
\begin{aligned}
\left\langle g v^{\prime}, v^{\prime}\right\rangle & =\lim _{n}\left\langle g v_{n}, v_{n}\right\rangle \\
& =\lim _{n}\left\langle g\left(u_{n}+v\right),\left(u_{n}+v\right)\right\rangle \\
& =\lim _{n}\left\langle g u_{n}, u_{n}\right\rangle+\lim _{n}\left\langle g u_{n}, v\right\rangle+\lim _{n}\left\langle g v, u_{n}\right\rangle+\lim _{n}\langle g v, v\rangle \\
& =\lim _{n}\left\langle g u_{n}, u_{n}\right\rangle+\langle g v, v\rangle .
\end{aligned}
$$

Since $\pi^{\prime}$ is irreducible, positive functions associated to it are extreme points in the cone of positive functions, a cone which is closed with respect to pointwise convergence (see, e.g., [1, Proposition C.5.2]). Therefore, $\lim _{n}\left\langle g u_{n}, u_{n}\right\rangle=t\left\langle g v^{\prime}, v^{\prime}\right\rangle$ for some $t \in \mathbb{R}$. In particular, $\left\langle g v^{\prime}, v^{\prime}\right\rangle=$ $\frac{1}{1-t}\langle g v, v\rangle(t \neq 1$ since $v \neq 0)$. The result follows by taking $\varphi=\sigma_{v /(t-1)}$.

\section{Additive Conjugacy of Irreducible Representations of $\mathbb{Z}$}

We would like to thank Yehuda Shalom for suggesting to us the results of this section.

The complex irreducible unitary representations of $\mathbb{Z}$ are one-dimensional, and given by multiplication by a complex number $z \in \mathbb{C}$ with $|z|=1$. We denote by $\pi^{z}$ the representation associated to $z$, so that for $n \in \mathbb{Z}$ and $x \in \mathbb{C}$ we have $\pi_{n}^{z} x=z^{n} x$.

Theorem 17. Two irreducible $\mathbb{Z}$ representations $\pi^{z}$ and $\pi^{w}$ are additive conjugates if and only if for every $p(x) \in \mathbb{Z}[x]$ it holds that $p(z)=0$ if and only if $p(w)=0$.

Proof. First suppose $\pi^{z}, \pi^{w}$ are additive conjugates, witnessed by an additive bijection $\xi$. Take $p(y) \in \mathbb{Z}[y]$ with $p(z)=0$. Write $p(y) \equiv r_{n} y^{n}+\cdots+$ $r_{1} y+r_{0}$. Take $x \in \mathbb{C}$ with $\xi(x) \neq 0$. Then

$$
\begin{aligned}
0 & =\xi\left(r_{n} z^{n} x+\cdots+r_{1} z x+r_{0} x\right) \\
& =r_{n}(w)^{n} \xi(x)+\cdots+r_{1} w \xi(x)+r_{0} \xi(x) \\
& =p(w) \xi(x),
\end{aligned}
$$

where the first equality is a consequence of $p(z)=0$, and the second follows from the additivity of $\xi$ and the fact that it intertwines $\pi^{z}$ and $\pi^{w}$. 
This gives $p(w)=0$. We have shown every $p(y) \in \mathbb{Z}[y]$ with $p(z)=0$ has $p(w)=0$. By symmetry, of course, we get the reverse.

Now suppose that for every $p(y) \in \mathbb{Z}[y]$, it holds that $p(z)=0$ if and only if $p(w)=0$. It is then well known that there is an isomorphism ${ }^{3}$ $\Xi: \mathbb{Q}(z) \rightarrow \mathbb{Q}(w)$ with $\Xi(z)=w$.

Let $\left\{x_{\alpha}\right\}_{\alpha}$ be a basis for $\mathbb{C}$ over $\mathbb{Q}(z)$ and $\left\{y_{\alpha}\right\}_{\alpha}$ be a basis for $\mathbb{C}$ over $\mathbb{Q}(w)$. Define $\xi: \mathbb{C} \rightarrow \mathbb{C}$ by

$$
\xi\left(\sum_{\alpha} c_{\alpha} x_{\alpha}\right)=\Xi\left(c_{\alpha}\right) y_{\alpha}
$$

which is well-defined (on all of $\mathbb{C}$ ) since $\left\{x_{\alpha}\right\}$ is a basis. Also, that $\Xi$ is a field isomorphism mapping $z$ to $w$ implies that $\xi$ is an additive bijection intertwining $\pi^{z}$ and $\pi^{w}$ :

$$
\xi\left(z^{n} \sum_{\alpha} c_{\alpha} x_{\alpha}\right)=\sum_{\alpha} \Xi\left(z^{n} c_{\alpha}\right) y_{\alpha}=\sum_{\alpha} w^{n} \Xi\left(c_{\alpha}\right) y_{\alpha}=w^{n} \xi\left(\sum_{\alpha} c_{\alpha} x_{\alpha}\right) .
$$

Corollary 18. The representations $\pi^{z}$ and $\pi^{w}$ are additive conjugates if and only if both $z, w$ are not algebraic numbers or they are both algebraic numbers and are Galois conjugates.

Note that it is easy to show that if, for example, $z$ and $w$ are not algebraic then $\pi^{z}$ and $\pi^{w}$ cannot be conjugate representations (in the usual sense) unless $z=w$. Thus this is an example of irreducible representations that are additive conjugates but not conjugates.

\section{REFERENCES}

[1] B. Bekka, P. de La Harpe, and A. Valette, Kazhdan's property (T), Vol. 11, Cambridge university press, 2008

[2] A. Connes and B. Weiss, Property $T$ and asymptotically invariant sequences, Israel Journal of Mathematics 37 (1980), no. 3, 209-210.

[3] E. Glasner and B. Weiss, Kazhdan's property T and the geometry of the collection of invariant measures, Geometric \& Functional Analysis GAFA 7 (1997), no. 5, 917935.

[4] P. R. Halmos, On automorphisms of compact groups, Bulletin of the American Mathematical Society 49 (1943), no. 8, 619-624.

\footnotetext{
${ }^{3}$ One can take $\Xi\left(\frac{p(z)}{q(z)}\right)=\frac{p(w)}{q(w)}$. It is easy to verify that this is a well-defined isomorphism.
} 
[5] E. Hewitt and K. A Ross, Abstract harmonic analysis: Volume i structure of topological groups integration theory group representations, Vol. 115, Springer Science \& Business Media, 2012.

[6] I. Kaplansky, Groups with representations of bounded degree, Canad. J. Math 1 (1949), 105-112.

[7] K. Schmidt, Amenability, Kazhdan's property T, strong ergodicity and invariant means for ergodic group-actions, Ergodic Theory and Dynamical Systems 1 (1981), no. $2,223-236$.

[8] _ From infinitely divisible representations to cohomological rigidity, Analysis, geometry and probability, 1996, pp. 173-197.

[9] _ Dynamical systems of algebraic origin, Springer Science \& Business Media, 2012.

California Institute of Technology, Pasadena, CA, 91125 